\title{
Exame papanicolau: percepção das mulheres sobre os motivos que influenciam a sua não realização
}

\author{
Pap smear: Perception of women about the reasons that influence their non-performance \\ Prueba papanicolaou: Percepción de las mujeres sobre los motivos que influyen en su no \\ realización
}

Iara Damascena Silva ${ }^{1 *}$, Maria Elizanete Teixeira da Silva ${ }^{1}$, Josimeire Souza de Oliveira Andrade ${ }^{1}$, Bianca Cristina Martins Nunes ${ }^{1}$, Carina Oliveira Pego'.

\section{RESUMO}

Objetivo: identificar os motivos para a não realização do exame Papanicolau por mulheres usuárias em uma UBS, em Porto Velho (RO). Métodos: Foi utilizado como método uma pesquisa qualitativa e descritiva com entrevista semiestruturada seguindo o roteiro com sete perguntas abertas e gravadas. Para análise de dados, utilizou-se como método a análise de conteúdo sendo considerados os aspectos éticos e legais da pesquisa determinados pela Resolução 466/12 do Conselho Nacional de Saúde/Ministério da Saúde. Participaram do estudo 20 de mulheres no período de janeiro à fevereiro de 2019. Notou-se que a baixa escolaridade é um fator influenciador no reconhecimento da importância do exame, a multivalência da mulher propicia o postergar de sua própria saúde e a falta de interesse é um fator que prevalente e impede a realização do exame. Conclusão: Percebemos que aos profissionais da USF cabem investir em reflexões sobre suas práticas e a busca de soluções que possam melhorar estrategicamente 0 atendimento e captação dessas mulheres, envolvendo atividades de educação em saúde para o fortalecimento das ações de prevenção e promoção voltadas ao público feminino.

Palavras-chave: Papanicolau, Câncer uterino, Adesão.

\begin{abstract}
Objective: to identify the reasons for not performing the Pap test pap smear by women users at a UBS, in Porto Velho (RO). Methods: A qualitative and descriptive research was used as a method with a semistructured interview following the script with seven open and recorded questions. For data analysis, the content analysis was used as a method, considering the ethical and legal aspects of the research determined by Resolution 466/12 of the National Health Council / Ministry of Health. Participants in the study were 20 of women from January to February 2019. It was noted that low level of schooling is an influential factor in the recognition of the importance of the exam, the multivalence of the woman propitiates the postponement of their own health and lack of interest is a factor that prevails and prevents the achievement of the examination. Conclusion: We realize that USF professionals can invest in reflections on their practices and the search for solutions that can strategically improve the care and recruitment of these women, involving health education activities to strengthen prevention and promotion actions aimed at the female public.
\end{abstract}

Key words: Pap smear, Uterine cancer, Adhesion.

\section{RESUMEN}

Objetivo: identificar los motivos para la no realización del examen Papanicolaou por mujeres usuarias en una UBS, en Porto Velho (RO). Métodos: Se utilizó como método una investigación cualitativa y descriptiva con

1Faculdade Interamericana de Porto Velho (UNIRON) Porto Velho/RO. *E-mail: iara.sivaloras@gmail.com 
entrevista semiestructurada siguiendo el guión con siete preguntas abiertas y grabadas. Para el análisis de datos, se utilizó como método el análisis de contenido propuesto por Minayo (2012), siendo considerados los aspectos éticos y legales de la investigación determinados por la Resolución 466/12 del Consejo Nacional de Salud / Ministerio de Salud. Participaron del estudio 20 de mujeres en el período de enero a febrero de 2019. Resultados: Se notó que la baja escolaridad es un factor influyente en el reconocimiento de la importancia del examen, la multivalencia de la mujer propicia el " postergar de su propia salud y la falta de interés es un factor que prevalece e impide la realización del examen. Conclusión: Percibimos que a los profesionales de la USF caben invertir en reflexiones sobre sus prácticas y la búsqueda de soluciones que puedan mejorar estratégicamente la atención y captación de esas mujeres, involucrando actividades de educación en salud para el fortalecimiento de las acciones de prevención y promoción dirigidas al público femenino.

Palabras clave: Papanicolaou, Cáncer uterino, Adhesión.

\section{INTRODUÇÃO}

O câncer do colo do útero tem um alto grau de morbidade e letalidade, porém apresenta possibilidade de cura se for diagnosticado precocemente. $O$ câncer do colo do útero é uma doença de evolução lenta, que apresenta fases detectáveis e curáveis. Mas ainda é um problema de saúde pública em países subdesenvolvidos ou em desenvolvimento, pois ainda apresenta altas taxas de prevalência e mortalidade em mulheres de baixa renda e que se encontra em plena fase reprodutiva. Dentre todos os cânceres existentes, o câncer do colo do útero é o que tem mais probabilidade de cura através da prevenção (LIMA M, et al., 2017).

O exame preventivo ou Papanicolau é um teste realizado para detectar a existência de alguma alteração celular do colo uterino. Este exame também é conhecido como esfregaço cérvicovaginal e colpocitologia oncótica cervical. O nome Papanicolau é uma homenagem ao criado do método Georges Papanicolau (BRASIL Ministério da Saúde, 2015).

Este exame pode ser feito em postos de saúde da rede pública desde que hajam profissionais qualificados. É a principal estratégia de diagnosticar lesões precocemente, antes mesmo que apareça algum sintoma patológico. O exame preventivo pode causar um pequeno incômodo, sendo ele indolor, simples e de rápida execução (BRASIL Ministério da Saúde, 2015).

Mesmo com a oferta nas unidades básicas de saúde, ainda existem mulheres que ainda possuem conhecimento limitado acerca da importância que o exame possui na prevenção do câncer do colo do útero e não o realizam regularmente (DIAS E, et al. 2015).

Apesar do Sistema Único de Saúde (SUS) ofertar na Unidade Básica de Saúde (UBS) recursos necessários para a prevenção do câncer de colo de útero, há um grande número de mulheres que não aderem a esta prática. Esta baixa adesão contribui negativamente na redução dos indicadores de sobrevida associados a esse tipo de câncer. Os fatores culturais, sociais, econômicos e comportamentais são considerados fatores determinantes para adesão e controle desse agravo (SILVA E, et al., 2015).

A Unidade de Saúde da Família (USF) é considerada o local oportuno para a realização de atividades educativas no controle do câncer do colo do útero, visto que é a porta de entrada das mulheres nos serviços de saúde. A relevância do enfermeiro no contexto da prevenção do câncer do colo do útero se dá pela orientação esclarecimento de dúvida, prevenção de fatores de risco, realização da consulta ginecológica e do exame preventivo do câncer do colo do útero assim sendo realiza uma melhor qualidade, efetivando um sistema de registro de qualidade e intervindo para o encaminhamento adequado (RAMOS A, et al., 2014).

No Brasil, a cada ano surgem 18 mil novos casos. Sendo a maior prevalência de câncer uterino na região Norte, que chega a ser o segundo tipo de neoplasia mais frequente, atrás apenas do câncer de mama, onde a cada 100 mil mulheres 22,8 são atingidas pelo câncer uterino e 16,6 pelo câncer de mama. Além da morbidade e mortalidade o câncer-uterino acarreta prejuízos socioeconômicos para a sociedade que incluem elevados custo do tratamento, redução da população economicamente ativa e consequências psicológicas e sociais pra as famílias das mulheres acometidas pela a doença (ANDRADE M, et al. 2014). 
O câncer de colo uterino apresenta altas taxas de prevalência e mortalidade em mulheres de nível social e econômico baixo e em fase produtiva de suas vidas, nos países em desenvolvimento. Estas, uma vez doentes, têm suas atividades laborais e familiares comprometidas, acarretando um prejuízo econômico-social de magnitude considerável (DANTAS P, et al., 2018).

Diante disto surge a questão norteadora: Quais os motivos que influenciam a não realização do exame Papanicolau?

Esta pesquisa tem como objetivo geral identificar os motivos para a não realização do exame Papanicolau por mulheres usuárias em uma UBS no município de Porto Velho-RO.

\section{MÉTODOS}

Estudo de campo, do tipo descritivo, exploratório, com abordagem qualitativa, com uso da técnica de entrevista com roteiro semi-estruturado, que foi realizado na USF Doutor José Adelino da Silva, localizada no setor leste do município de Porto Velho-RO.

O local escolhido para esta pesquisa foi USF Doutor José Avelino Silva, unidade de saúde gerida pela Secretaria Municipal de Saúde (SEMUSA) de Porto Velho, localizado na cidade de Porto Velho, Rondônia. Esta unidade é formada por cinco equipes de Estratégia Saúde da Família (ESF), composta por cinco enfermeiros, nove técnicos de enfermagem, cinco odontólogos, cinco médicos da unidade e mais dois médicos diarista que atendem nas segundas e quartas feiras e vinte e cinco agentes comunitário de saúde. Nesta unidade existe laboratório, sala de vacina e pequenos procedimentos como curativos e retiradas de pontos cirúrgicos.

A pesquisa constituiu-se de mulheres que já foram atendidas na USF Doutor José Adelino da Silva, mas que nos últimos cinco anos não realizaram o exame Papanicolau. Das pacientes atendidas que não realizaram o exame, foi feito um sorteio, sendo selecionadas por livre demanda as pacientes de 18 a 60 anos que se enquadraram nos objetivos da pesquisa.

A amostra foi composta por 20 mulheres que se encaixaram no pré-requisito de não terem realizado o exame Papanicolau nos últimos cinco anos ou nunca terem realizado o mesmo, que aceitaram participar voluntariamente do estudo.

A pesquisa iniciou-se após a autorização institucional da prefeitura do município de porto Velho-SEMUSA, com anuência no dia 26 de novembro de 2018 e aprovação do Comitê de Ética e Pesquisa (CEP) da União Educacional do Norte Ltda - UNINORTE no dia 19 de dezembro de 2018 com número de CAAE: 98408718.0.0000.8028.

Conforme estabelecido na Resolução no 466, de 12 de dezembro de 2012, do Ministério da Saúde, toda pesquisa que envolve seres humanos e informações pessoais, estes dados serão mantidos em total sigilo e confidencialidade, e serão de uso exclusivo de atender aos objetivos desta pesquisa, preservando o anonimato das informações. Ainda de acordo com a Resolução no 466/12, toda e qualquer pesquisa pode oferecer riscos para os seus colaboradores, sendo estes riscos variáveis conforme os objetivos do estudo. A pesquisa foi iniciada somente após a aprovação deste projeto junto ao Comitê de Ética e Pesquisa (CEP).

Os resultados divulgados e publicados de forma a contribuir para a melhoria na assistência (mulheres), pautando-se sempre na preservação da imagem da população que compõe a amostra desta pesquisa, de forma a evitar a exposição de imagem das mesmas. A pesquisa também considerou os aspectos éticos presentes na Resolução n 510, de 7 de abril de 2016, do Conselho Nacional de Saúde.

Foi entregue o Termo de Consentimento Livre e Esclarecido (TCLE) ao pesquisado, com a descrição dos objetivos da pesquisa e o livre arbítrio para negar a responder ou manifestar sua vontade de não mais participar da pesquisa, bem como também a necessidade da assinatura do mesmo. Ressalva-se, quanto o participante da pesquisa, rubricaram todas as folhas do TCLE, pondo sua assinatura na última página do referido Termo. 


\section{RESULTADOS E DISCUSSÃO}

Participaram do estudo 20 mulheres que atenderam aos critérios de inclusão e exclusão da pesquisa. Como critérios de inclusão, foram convidadas a participar do estudo as mulheres de 18 a 60 anos que já foram atendidas na unidade pesquisada e que não realizaram o Papanicolaou nos últimos cinco anos, assim como aquelas que concordarem em participar da pesquisa por meio da assinatura do Termo de Consentimento Livre e Esclarecido (TCLE).

Para os critérios de exclusão: não compuseram a amostra do estudo aquelas mulheres que não fazem acompanhamento na unidade, as que possuam dificuldade de compressão do TCLE, as gravações inaudíveis e as que se negarem a participar da pesquisa.

$\mathrm{Na}$ caracterização das participantes da pesquisa, foi possível identificar que a faixa etária predominante foi de 20 a 30 anos, porém de 31 a 40 anos foi semelhante.

A partir da análise das falas, tendo como base relatos semelhantes e convergentes, foram elencados três núcleos temáticos: escolaridade como fator influenciador na realização do exame Papanicolau, a mulher multivalente e a realização do exame Papanicolau e o desinteresse com o autocuidado.

\section{Escolaridade como fator influenciador na realização do exame Papanicolau}

Conforme os relatos das entrevistadas, pode se perceber que a baixa escolaridade influência na forma de se expressar e entender as perguntas, por desconhecerem os significados de algumas palavras ficavam mais introvertida ao responderem as perguntas, para algumas o nome Papanicolau era novo, pois conhecia 0 exame por exame preventivo.

O nível de escolaridade das entrevistadas é considerável insatisfatório, já que assuntos que correspondem à educação sexual também são debatidos no âmbito escolar. A baixa escolaridade pode também estar associada ao baixo poder aquisitivo das participantes do estudo. O nível de escolaridade certamente afeta a compreensão das mulheres sobre a gravidade do câncer de colo de útero.

Souza E et al., 2013 e Silva et al., 2019, relatam que as mulheres que apresentam baixa escolaridade têm maior probabilidade de não realizar o exame Papanicolau, tendo como barreira a dificuldade a compreensão e entendimento sobre o câncer do colo do útero e do próprio exame Papanicolau.

\section{A mulher multivalente e a realização do exame Papanicolau}

A mulher nos últimos anos vem ocupando mais espaço no mercado de trabalho, assim como se tornando a mantedora dos seus lares, isso faz com que as mulheres negligenciem sua própria saúde, priorizando o financeiro e o bem-estar familiar. $O$ atendimento nas unidades de saúde tem horários fixos e dias específicos para realização dos exames, não sendo adequados à rotina da mulher atuante no mercado de trabalho que se torna dependente da liberação do trabalho (SOLDATELLI B, et al., 2016).

De acordo com a organização Mundial de Saúde (OMS), as estratégias para a detecção precoce de mulheres com sinais e/ou sintomas da doença, o rastreamento deve ser através da aplicação de um teste ou exame numa população assintomática, aparentemente saudável, com objetivo de identificar lesões sugestivas de câncer e encaminhá-la para investigação e tratamento. Quando diagnosticado e tratado precocemente, constitui uma causa de morte evitável, pois esta neoplasia apresenta etapas bem definidas, com longo período para a evolução das lesões. Sabe-se que o Papanicolau é o exame que confere um dos mais altos potenciais de prevenção e cura entre todos os tipos de câncer (LIMA H, 2019). Com a crise econômica que se instalou na última década, tornou-se mais recorrente a existência de barreiras físicas e estruturais que dificultam o acesso e a procura da população pelos serviços de saúde, sobretudo na rede básica, isso tem comprometido, a integralização do cuidado e, principalmente o acesso e a qualidade da atenção (AMORIM L, et al., 2018).

\section{$O$ desinteresse com o autocuidado}

Mesmo com a oferta do exame Papanicolau nas unidades básicas de saúde e unidade de saúde da família, com campanhas existentes voltadas a saúde da mulher, a falta de interesse e um fator de considerável 
relevância, durante a nossa pesquisa pode-se perceber que a falta de interesse estar relacionada a falta de conhecimento das mulheres sobre a necessidade da realização do exame Papanicolau para a prevenção do câncer do colo do útero. As falas abaixo revelam a falta de interesse na realização do exame, fator fundamental para quebra de paradigmas. Quando perguntadas qual o motivo de não realizarem o exame Papanicolau as respostas foram diretas.

\section{"Por que não quis" \\ "Não me interessei mesmo"}

O resultado se contrapõe com algumas pesquisas, que apresentam um menor índice aos resultados da falta de interesse. Nos estudos de Costa D et al., (2018) envolvendo 35 mulheres, cinco relataram que não achavam necessária a realização do exame, já que não apresentavam nenhum sintoma aparente. A falta de interesse ou descuido foi o discurso de quatro mulheres, os relatos mais presentes durante a realização de sua pesquisa.

Entre os problemas encontrados pelas mulheres para a realização do Papanicolau, a falta da oferta de informação por parte dos profissionais de saúde se torna um agravante na busca da realização do exame. Percebe-se que o trabalho em grupo proporciona conhecimento e desperta o interesse no autocuidado, sendo que a troca de experiências permite que os participantes esclareçam dúvidas, compartilhem medos, e principalmente se sintam motivadas a realizar o exame (LIMA H, et al., 2019).

Segundo Lima H, et al., (2019), a enfermagem é quem trabalha diretamente com o exame Papanicolau e, assim, é a grande responsável pelo repasse das informações sobre este exame e tantos outros. Assim, a enfermagem acaba se tornando a responsável pela educação e saúde da população, pelo o repasse das informações, utilizando de tecnologias leves na execução da educação e saúde.

A enfermagem se destaca na tarefa do cuidado preventivo, desenvolvendo novas estratégias motivacionais e que mobilizem os profissionais envolvidos na realização da prevenção. Uma das formas é orientar sobre a importância da realização de exames preventivos de forma interativa, promovendo 0 autoconhecimento e confiança (SILVA E, et al., 2010).

Cabe aos profissionais de saúde influenciar as práticas de cuidado, sem imposição de condutas e assim recrutar mulheres para realização de ações educativas de maneira dialogada. É preciso prevalecer o respeito e $o$ atendimento integral e igualitário (ACOSTA D, et al., 2017).

\section{CONSIDERAÇÕES FINAIS}

A realização desta pesquisa nos permitiu a compreensão e a reflexão sobre as dificuldades verbalizadas que as impediam de realizar o exame Papanicolau. Dentre estas dificuldades, foi observado que a falta de conhecimento é um fator preponderante para a não realização do exame, mas que a falta de escolaridade é um fator importante e as multi responsabilidades da mulher interfere na realização do exame Papanicolau, $e$ diante desses aspectos, aos profissionais da USF cabem investir em reflexões sobre suas práticas e a busca de soluções que possam melhorar estrategicamente o atendimento e captação dessas mulheres, envolvendo atividades de educação em saúde para o fortalecimento das ações de prevenção e promoção voltadas ao público feminino.

\section{REFERÊNCIAS}

1. ACOSTA D, DANTAS T, CAZEIRO C et al. Vivenciando o exame papanicolau: entre o (não) querer e o fazer. Rev enferm UFPE online., Recife, 11(8): 3031-8, ago., 2017.

2. AMORIM L, MONTEIRO N, NOGUEIRA L et al. Exame de colpocitologia oncótica: revisão integrativa pap smear exam: integrative review examen de colpocitologia oncótica: revisión integradora. Rev Enferm Health Care [online]. Jan/Jul 2018; 7(1):209-224.

3. ANDRADE M, ALMEIDA M, ARAUJO T et al. Fatores associados à não adesão ao Papanicolau entre mulheres atendidas pela Estratégia Saúde da Família em Feira de Santana, Bahia, 2010. Epidemiol. Serv. Saúde, Brasília, jan-mar 2014. 
4. BRASIL, Ministério da saúde. Biblioteca virtual em saúde. Papanicolau (exame preventivo do colo do útero setembro de 2015.

5. DANTAS P, LEITE K, CÉSAR E et al., Conhecimento das mulheres e fatores da não adesão acerca do exame papanicolau. Rev enferm UFPE online., Recife, 12(3):684-91, mar., 2018.

6. DIAS E, SANTOS D, DIAS ZN et al. Perfil socioeconômico e prática do exame de prevenção do câncer do colo do útero de mulheres de uma unidade de saúde. Revista Saúde e Desenvolvimento vol. 7, n.4 | jan - dez 2015.

7. LIMA HF. Importância do exame papanicolau na gestação: uma revisão de literatura. Mostra Interdisciplinar do curso de Enfermagem, [S.I.], mar. 2019 CLEMENT S, SHELFORD VE. Bio-ecology: an introduction. 2nd ed. New York: J. Willey, 1966; 425p.

8. LIMA M, PALMEIRA M, MENEZES $P$ et al. Motivos que influenciam a não-realização do exame de papanicolaou segundo a percepção de mulheres. Temas em saúde Volume 17, Número 1 ISSN 2447-2131 João Pessoa, 2017.

9. MINAYO, M.C. S. (2012). Análise qualitativa: teoria, passos e fidedignidade. Ciência \& Saúde Coletiva,17(3), 621--626.

10. RAMOS A, SILVA D, MACHADO G et al. 2014. A atuação do enfermeiro da estratégia saúde da família na prevenção do câncer de colo de útero. S a $n$ a r e, Sobral, V.13, n.1, p.84-91, jan./jun. - 2014

11. SILVA E, DIAS M, FERNANDES C et al. 2015. Fatores relacionados a não adesão à realização do exame de Papanicolau. Rev Rene. 2015 jul-ago.

12. SOLDATELLI BE, VIGO AL, GIUGLIANI ERJ et al. Adherence to dietary recommendations for preschoolers: clinical trial with teenage mothers. Revista de Saúde Pública, São Paulo, v. 50, p. 83, jan. 2016. ISSN 15188787. 